Journal of Animal and Veterinary Advances 11 (9): 1353-1355, 2012

ISSN: $1680-5593$

(C) Medwell Journals, 2012

\title{
Tissue Expression Level Analysis of CD14 in Buffalo
}

\author{
Donglin Zhang, Yongchang Hao, Ying Cheng, Li Du, Ming Lei, Hanwei Jiao, Jianing Zhang, \\ Hui Rong, Xiaoxiao Jia and Fengyang Wang \\ Hainan Key Lab of Tropical Animal Reproduction and Breeding and Epidemic Disease Research \\ (Construction Period), Animal Genetic Engineering Key Lab of Haikou, College of Agriculture, \\ Hainan University, 570228 Haikou, People's Republic of China
}

\begin{abstract}
Brucellosis is an extremely important disease affecting the health of human and a number of animal species (including buffalo) around the world and Brucellae sp. is the causative agent of brucellosis. As an important innate immunity molecule, CD14, involved in protecting a host against invading microorganism including Brucella. To analyze the expression of CD14 and provide new alternatives for elucidating the molecular mechanism of Brucella infection in bufflo, real-time quantitative Reverse Transcription PCR (RT-PCR) and western blot were performed to assess the mRNA level and protein levels of CD14 in heart, liver, spleen, lung, kidney and intestine. The results indicated that CD14 mRNA and protein are ubiquitously present in all buffalo tissues examined with relatively high levels in lung and relatively low levels in intestine. These data provide important anatomical information for studying the role of CD14 played in Brucella infection in the different tissue of buffalo.
\end{abstract}

Key words: Buffalo, CD14, real-time RT-PCR, Western blot, tissue expression level, China

\section{INTRODUCTION}

Brucellae sp. is the causative agent of brucellosis, a disease of worldwide importance and affects human and a number of animal species (Abd El-Razik et al., 2007a, b; Pakzad et al., 2010; Hawari, 2012). The six traditional species are $B$. melitensis, B. suis, B. abortus, B. neotomae, $B$. ovis and $B$. canis. Despite the fact that the first member of the genus was described $>100$ years ago, the virulence mechanisms of Brucella are not very clear, especially in buffalo (Corbel, 1997).

As an important virulence determinant of Brucella, the Lipopolysaccharide (LPS) in the outer membrane binds to lipopolysaccharide-binding protein (a serum protein) which in turn transfers it to the blood phase of CD14. The CD14-LPS complex binds to Toll like receptor proteins on the surface of macrophage cells triggering the release of proinflammatory cytokines (Hirsh et al., 2004). And as the receptor of LPS, CD14, occurs as a 55 kilodaltons ( $\mathrm{kDa}$ ) Glycosyl Phosphatidylinositol (GPI) anchored glycoprotein attached to the membrane in myeloid cell lines or a soluble form in serum lacking the GPI-anchor (Avni et al., 2010; Gomes et al., 2010; Triantafilou et al., 2000). In addition to its role as an LPS receptor, other functions of CD14 have been noted. It facilitates attachment of monocytes to activated endothelial cells and to activated $\mathrm{T}$ cells (Beekhuizen et al., 1991).

Although, the findings on the expression levels of CD14 in different tissues are able to provide important anatomical clues for evaluating the role of CD14 played in the interaction between Brucella and target cells in the different tissue of animal, there are few reports about its expression level analysis in normal tissues of animal, especially in buffalo. In this report, to investigate the presence of CD14 in the tissues of buffalo, real-time quantitative RT-PCR and western blot were performed to assess the expression levels of CD14 in heart, liver, spleen, lung, kidney and intestine, respectively. The findings suggest that CD14 are ubiquitously present in heart, liver, spleen, lung, kidney and intestine of buffalo and the protein expression level in lung is the highest, the protein expression level in intestine is the lowest which provide important anatomical information for elucidating the role of CD14 played in Brucella infection in buffalo.

\section{MATERIALS AND METHODS}

Buffalo tissues: Adult Xinglong buffalos, a local buffalo breed were sacrificed and heart, liver, spleen, lung, kidney

Corresponding Author: Fengyang Wang, Hainan Key Lab of Tropical Animal Reproduction and Breeding and Epidemic Disease Research (Construction Period), Animal Genetic Engineering Key Lab of Haikou, College of Agriculture, Hainan University, 570228 Haikou, People's Republic of China 
and intestine tissue were obtained immediately. The tissues used for real time RT-PCR and western blot analyses were initially immersed in liquid nitrogen and preserved at $-70^{\circ} \mathrm{C}$. All animal experimental protocols complied with national legislation and company policy on the care and use of animals according to the related code of practice.

Real-time quantitative RT-PCR: The PureLink ${ }^{\mathrm{TM}}$ RNA Mini kit (Invitrogen, USA) was used to isolate highquality total RNA from buffalo tissues according to the manufacturer's instructions. The concentration and integrity of purified RNA were measured by absorption of light at 260 and $280 \mathrm{~nm}$ (A260/280) and electrophoresis after staining with ethidium bromide.

Synthesis of cDNA and determining of expression levels of CD14 mRNA were performed as described previously (Hao et al., 2012). The comparative CT Method was used to compare the CD14 mRNA levels of different tissues. To control variability of RNA input, all PCR reactions were normalized to the $\mathrm{Ct}$ value of $28 \mathrm{~S}$ rRNA of buffalo. Each assay represented three independent experiments and within an experiment, all samples were performed in triplicate. All data are expressed as mean $\pm \mathrm{SD}$. The sequence of the primers for detection of CD14 and 28S rRNA were as follows:

CD14-F: 5'-GACCTGTCTGACAATCCCAGTCT-3', CD14-R: 5'-TTGCGTAGCGCTAGATATTGGA-3' 28S rRNA-F: 5'-GTGCTTAGTGGGCTACTTTTGGTAAG-3' 28S rRNA-R: 5'-TGCTGTCTATATCAACCAACACATTTT-3'

Western blot: Western blot analyses were performed as described previously (Hao et al., 2012). Primary antibodies consisted of rabbit polyclonal anti-bovine CD14 (1:1000) (Abcam, USA) and GAPDH rabbit mAB (1:1200) (cell signaling technology, USA), respectively. Secondary antibodies were HRP labeled goat anti-rabbit IgG (1:4000) (Santa Cruz Biotechnology, USA). After incubation with the secondary antibodies for $1 \mathrm{~h}$ at $37^{\circ} \mathrm{C}$, membranes were developed using an ECM kit (Boster, China) for $5 \mathrm{~min}$ and detected by Charge-Coupled Device (CCD) cameras.

\section{RESULTS AND DISCUSSION}

Brucella organisms are intracellular parasites of mammals including buffalo. In vivo, Brucella has been described within bovine, caprine and murine trophoblasts, in caprine lymphocytes, $M$ cells, chicken embryo fibroblasts as well as a number of professional phagocytes lining different tissues (Holland and Picektt, 1956; Richardson and Holt, 1964; Anderson and Cheville,

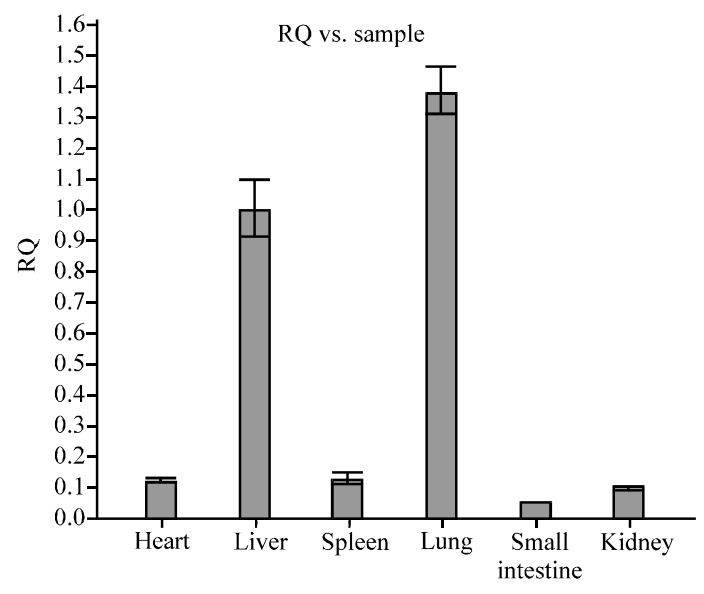

Fig. 1: Relative expression levels of CD14 mRNA in buffalo heart, liver, lung, spleen, kidney and intestine tissues

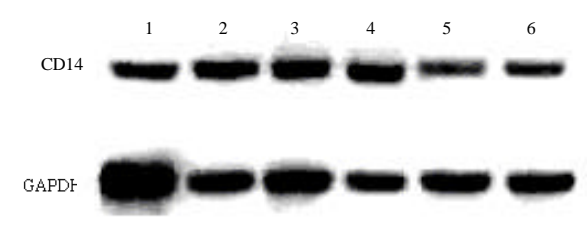

Fig. 2: Western blot analysis of CD14 protein expression in buffalo heart, liver, lung, spleen, kidney and intestine tissues

1986; Anderson et al., 1986; Ackermann et al., 1988 Detilleux et al., 1988; Cheville et al., 1996). Analysis and identification of molecules in the host cells in different tissues involved in the invasion process and trafficking, like CD14 are necessary to understand the events leading to the establishment of the infection.

Based on the previous study in which the complete sequences of buffalo CD14 cDNA were cloned and deposited into GENEBANK (Accession No.: HM749882), real-time RT-PCR analyses were performed, the results indicated that CD1 4 mRNA were ubiquitously present in all buffalo tissues examined. Further the mRNA level observed are listed as follows from high to low: lung, liver, spleen, heart, kidney and intestine (Fig. 1).

To further determine the expression level of CD14 protein in all buffalo tissues examined and provide important anatomical information on the role of CD14 in the interaction between Brucella and target cells in the different buffalo tissue, Western blot were performed, the results indicated that the CD14 protein were expressed in all buffalo tissues examined and the protein expression level in lung is the highest, the protein expression level in intestine is the lowest which are keeping with the results of real-time RT-PCR (Fig. 2). 
The study showed that CD14 mRNA and protein are ubiquitously present in all buffalo tissues examined with relatively high levels in lung and relatively low levels in intestine. These data suggested that it is possible that CD14 may play more important function in lung than that in other tissues examined. CD14 mRNA and protein levels in lung, kidney, liver, heart and intestine were different indicating that the regulation of expression in these tissues is altered and likely to be correlated with its role in the interaction between Brucella and target cells in the different tissue of buffalo.

\section{CONCLUSION}

The findings provide important anatomical information for studying the role of CD14 played in Brucella infection in the different tissue of buffalo. Further studies are likely to include investigating the interaction of CD14 in buffalo tissues with important Brucella bacterial proteins.

\section{ACKNOWLEDGEMENTS}

This study was financially supported by the Major National Science and Technology Specific Projects (2009ZX08007-009B) and the National High Technology Research and Development Program of China (2011AA100302). Dongling Zhang and Yongchang Hao contributed equally to this research.

\section{REFERENCES}

Abd El-Razik, K.A., H.M. Desoukey and W.M. Ahmed, 2007b. Investigations on brucellosis in Egyptian baladi does with emphasis on evaluation of diagnostic techniques. Pak. J. Biol. Sci., 10: 342-348.

Abd El-Razik, K.A., Y.A. Ghazi and E.M. Salama, 2007a. Monitoring of brucella reactor does following milk examination using different techniques. Pak. J. Biol. Sci., 10: 240-244.

Ackermann, M.R., N.F. Cheville and B.L. Deyoe, 1988. Bovine ileal dome lymphoepithelial cells: Endocytosis and transport of Brucella abortus strain 19. Vet. Pathol., 25: 28-35.

Anderson, T.D. and N.F. Cheville, 1986. Ultrastructural morphometric analysis of Brucella abortus-infected trophoblasts in experimental placentitis. Bacterial replication occurs in rough endoplamic reticulum. Am. J. Pathol., 124: 226-237.

Anderson, T.D., N.F. Cheville and V.P. Meador, 1986. Pathogenisis of placentitis in the goat inoculated with Brucella abortus II. Ultrastructural studies. Vet. Pathol., 23: 227-239.
Avni, D., O. Ernst, A. Philosoph and T. Zor, 2010. Role of CREB in modulation of TNF $\alpha$ and IL-10 expression in LPS-stimulated RAW264.7 macrophages. Mol. Immunol., 47: 1396-1403.

Beekhuizen, H., I. Blokland, T.A.J. Corsel-Van, F. Koning and R. van Furth, 1991. CD14 contributes to the adherence of human monocytes to cytokinestimulated endothelial cells. J. Immunol., 147: $3761-3767$.

Cheville, N.F., S.C. Olsen, A.E. Jensen, M.G. Stevens and A.M. Florance et al., 1996. Bacterial persistence and immunity in goats vaccinated with a purE deletion mutant or the parental $16 \mathrm{M}$ strain of Brucella melitensis. Infect. Immun., 64: 2431-2439.

Corbel, M.J., 1997. Brucellosis: An overview. Emerg. Infect. Dis., 3: 213-221.

Detilleux, P.G., N.F. Cheville and B.L. Deyoe, 1988. Pathogenesis of Brucella abortus in chicken embryos. Vet. Pathol., 25: 138-146.

Gomes, N.E., M.K. Brunialti, M.E. Mendes, M. Freudenberg, C. Galanos and R. Salomao, 2010. Lipopolysaccharide-induced expression of cell surface receptors and cell activation of neutrophils and monocytes in whole human blood. Braz. J. Med. Biol. Res., 43: 853-858.

Hao, Y.C., X.R. Zhang, D.L. Zhang, Y. Cheng and L. Du et al., 2012. Molecular cloning and tissue expression of cell division cycle 42 in Buffalo tissue. J. Anim. Vet. Adv., (In Press).

Hawari, A.D., 2012. Epidemiological studies, seroprevalance and some risk factors of brucellosis in sheep and goats in the South Province of West Bank. Asian J. Animal Vet. Adv., 7: 535-539.

Hirsh, D.C., N.J. Maclachlal and R.L. Walker, 2004. Veterinary Microbiology. 2nd Edn., Wiley-Blackwell, USA., ISBN: 9780813803791, pp: 225-228.

Holland, J.J. and M.J. Picektt, 1956. Intracellular behavior of Brucella variants in chick embryo cells in tissue culture. Proc. Soc. Exp. Biol. Med., 93: 476-479.

Pakzad, I., A. Rezaee, M.J. Rasaee, A.Z. Hosseini and B. Tabbaraee et al., 2010. Novel approach of vaccination against brucella abortus 544 based on a combination of fusion proteins, human serum albumin and brucella abortus lipopolysaccharides. J. Biol. Sci., 10: 767-772.

Richardson, M. and J.N. Holt, 1964. Multiplication of Brucella in cultured lymphoid and non-lymphoid cells. J. Bacteriol., 88: 1163-1168.

Triantafilou, M., K. Triantafilou and N. Fernandez, 2000. Rough and smooth forms of fluorescein-labelled bacterial endotoxin exhibit CD14/LBP dependent and independent binding that is influenced by endotoxin concentration. Eur. J. Biochem., 267: 2218-2226. 